\title{
A density-division embedding potential inversion technique
}

\author{
O. Roncero, ${ }^{1, a)}$ A. Zanchet, ${ }^{1}$ P. Villarreal, ${ }^{1}$ and A. Aguado ${ }^{2}$ \\ ${ }^{1}$ Instituto de Física Fundamental, C.S.I.C., Unidad Asociada UAM-CSIC, Serrano 123, \\ 28006 Madrid, Spain. \\ ${ }^{2}$ Departamento de Química Física, Unidad Asociada UAM-CSIC, Facultad de Ciencias C-XIV, \\ Universidad Autónoma de Madrid, 28049 Madrid, Spain
}

(Received 9 October 2009; accepted 25 November 2009; published online 18 December 2009)

\begin{abstract}
A new method is proposed to partition the density of a system in two portions. The density on each subsystem is the solution of a Fock equation modified by the addition of an embedding potential. This embedding potential is obtained iteratively by minimizing the difference between the electronic densities of the total system and the sum of the subsystems. Thus, the electronic density partition and the embedding potential are obtained at the same time within the procedure, guarantying the v-representability of the densities partitioned. This fact is a considerable improvement of a recently proposed embedding potential inversion technique, [O. Roncero, M. P. de Lara-Castells, P. Villarreal, F. Flores, J. Ortega, M. Paniagua, and A. Aguado, J. Chem. Phys. 129, 184104 (2008)], in which the embedding potential is obtained once the electronic density is previously partitioned. The method is first applied to a linear $\mathrm{H}_{10}$ chain to illustrate how it works. The orbitals obtained are localized on each subsystem, and can be used to include local electronic correlation with currently available $a b$ initio programs. Finally, the method is applied to include the electronic correlation needed to describe the van der Waals interaction between $\mathrm{H}_{10}$ chains and $\mathrm{H}_{2}$ molecules, of $\approx 12$ meV, giving very accurate results. (C) 2009 American Institute of Physics. [doi:10.1063/1.3274823]
\end{abstract}

\section{INTRODUCTION}

One challenge in molecular physics is the theoretical description of large systems, requiring the calculation not only of potential energy surfaces but also of the dynamics at finite temperatures. The complete description of the system is generally not affordable. Very often, however, the process of interest occurs in a localized region of the whole system, making possible to consider separately this active region (A) from the environment (B). In such cases, different approximations can be used for the two regions, combining a high level method for A with a low level method for B. The coupling between the two subsystems can be treated in many different ways, from entirely quantum schemes to diverse combinations of molecular dynamics and quantum approaches. $^{1-3}$

The effect of the environment B on the primary part (A) is generally taken into account using a mean field approach through the so-called embedding potential (EP). In some situations, where the process is very well localized as in covalent insulating systems, a cluster approach is well adapted, in which subsystem $\mathrm{A}$ is separated from the whole system adding some atoms at its edges to saturate bonds. ${ }^{4-7}$ In ionic crystals, the cluster is embedded in the background formed by the charges of the surroundings, described by core pseudopotentials for cations, or point charges for anions, and account for long-range interactions. ${ }^{8-11}$ In metalliclike systems, however, the electronic density is much more delocalized and more refined methods must be applied. The EPs

${ }^{a)}$ Electronic mail: oroncero@imaff.cfmac.csic.es. calculated using density functional theory (DFT) techniques provide a natural improvement to describe the interaction of a subsystem and its environment. ${ }^{12-14}$

A very successful method is the so-called frozen density embedding (FDE) approach of Wesolowski and Warshel. ${ }^{14}$ In this method, the density of the whole system and that of the active cluster $\mathrm{A}$ is first obtained from DFT calculations, and the EP is derived from them iteratively. This method has been applied to many large systems using DFT-in-DFT techniques and different approaches for the environment. ${ }^{14-17}$ In addition, an intensive work has been devoted to improve this method for treating electronic correlation using multideterminantal wave functions ${ }^{18}$ and analyzing the effect of different functionals used for the kinetic energy term. ${ }^{19}$

A refinement of the FDE method has been recently developed by Carter and co-workers ${ }^{20-23}$ to treat the interaction of molecules with periodic metal surfaces. In this method, the EP is first obtained following the FDE approach of Wesolowski and Warshel ${ }^{14}$ using DFT methods. The EP is then added to the Fock operator leading to a modified HartreeFock (MHF) equation, which is solved to obtain the molecular orbitals, localized in subsystem A. With these orbitals any conventional $a b$ initio calculation can be performed, including electron correlation effects, opening the possibility of calculating excited electronic states.

One difficulty of the FDE approach is related to the kinetic energy functional used and has been the subject of an intensive analysis. ${ }^{19-22,24}$ Very recently, an alternative inversion method for obtaining the EP has been proposed, the EP inversion method (EPI). ${ }^{25}$ This method consists of three steps. First, the density of the total system is partitioned between $\mathrm{A}$ and $\mathrm{B}$. The density in $\mathrm{A}, \rho_{\mathrm{A}}$, is then considered as 
the reference density. Second, an EP $\mathcal{V}^{k}$ is added to the Fock operator of subsystem A, so that the solutions of the MHF equation yields a new density $\rho_{\mathrm{A}}^{k}$. The EP $\mathcal{V}^{k}$ is determined iteratively by minimizing the $\rho_{\mathrm{A}}-\rho_{\mathrm{A}}^{k}$ difference. In this inversion procedure a self-interaction potential is used, defined as the Coulomb interaction due to the density difference $\Delta_{\mathrm{A}}^{k}=\rho_{\mathrm{A}}-\rho_{\mathrm{A}}^{k}$ at each iteration, as proposed by Zhao, Morrison, and Parr. ${ }^{26}$ The final step of the method consists in using the confined orbitals in subsystem $\mathrm{A}$ in standard $a b$ initio methods to introduce electronic correlation, as done by Carter and co-workers. ${ }^{20-23}$

This EPI method is closely related to those devoted to extract (or invert) the exchange correlation potential from the electronic density obtained with an exact method, ${ }^{26-34}$ a subject of increasing activity.

The major problem of the EPI method, however, is the initial partition of the density. In Ref. 25, this problem was not directly addressed. Instead, a simple Mulliken partition method was used for the model case of linear hydrogen chains. This kind of separation is very crude but it was considered reasonable because it treats democratically all the atoms which are similar. However, such partition method presents several problems. It is quite arbitrary, and it is not guaranteed that the resulting $\rho_{\mathrm{A}}$ density is v-representable.

The aim of this work is to address the initial division of the total density. For this purpose a new method is developed, hereafter called density division plus embedding potential inversion (DDEPI) method, which is described in detail in Sec. II. In Sec. III, this method is applied to hydrogen chain $\mathrm{H}_{10}$ to illustrate how it works. Also this method is applied to study the interaction between $\mathrm{H}_{10}$ and a $\mathrm{H}_{2}$ molecule. This, weak van der Waals interaction is difficult to describe using conventional DFT techniques, and constitutes a benchmark case. ${ }^{25}$ Finally, Sec. IV is devoted to conclusions.

\section{DDEPI METHOD}

The aim of this work is to develop a method in which electronic correlation is introduced in subsystem A, whenever it is computationally impossible to treat it for the whole system. Thus, $\rho$ corresponds to the density of the whole system calculated with a monodeterminantal method, either HF of DFT. Note that the method described below to partition the density is rather general and could be applied for a density obtained with a more correlated multideterminantal method. In the present method, $\rho$ is iteratively partitioned as

$$
\rho=\rho_{\mathrm{A}}^{k}+\rho_{\mathrm{B}}^{k},
$$

where $k$ denotes the iteration and $\mathrm{A}$ and $\mathrm{B}$ to each of the subsystems. The iterative procedure corresponds to the minimization of the density difference

$$
\Delta^{k}=\rho-\left(\rho_{\mathrm{A}}^{k}+\rho_{\mathrm{B}}^{k}\right) .
$$

Since the density difference $\Delta^{k}$ can be positive and negative, it is convenient to work with its square as a measure of the real density difference. Such quantity is defined $\mathrm{as}^{25,31}$

$$
\delta^{k}=\int d \mathbf{r}\left(\Delta^{k}\right)^{2}=\operatorname{Tr}\left[\mathbf{S} \Delta^{k} \mathbf{S} \Delta^{k}\right],
$$

where $\mathbf{S}$ is the overlap matrix. Thus, the quantity to be minimized is $\delta^{k}$.

In this procedure $\rho_{\mathrm{A}}^{k}$ and $\rho_{\mathrm{B}}^{k}$ are solutions of a MHF equation,

$$
\left[\hat{\mathcal{F}}_{C}+\hat{\mathcal{V}}_{C}^{k}\right] \phi_{C, l}^{k}=\epsilon_{C, l}^{k} \phi_{C, l}^{k},
$$

where $C=\mathrm{A}$ or B denotes the subsystem, and $l$ the molecular orbital. $\hat{\mathcal{F}}_{C}$ is the Fock operator and $\mathcal{V}_{C}^{k}$ are the EPs at iteration $k . \rho_{\mathrm{A}}^{k}$ and $\rho_{\mathrm{B}}^{k}$ are by construction v-representable since they are obtained from the orbitals $\phi_{C, l}^{k}$ in Eq. (4). Note that this MHF equation is formally equivalent to that used in a Kohn-Sham method provided that the exchange term is treated exactly, and considering the EP $\mathcal{V}$ as a sort of correlation term with the environment.

The EPs are obtained iteratively as

$$
\mathcal{V}_{C}^{k+1}=\mathcal{V}_{C}^{k}+\lambda v^{k}\left[\Delta^{k}\right],
$$

where $\lambda$ is a parameter which minimizes $\delta^{k}$ at each iteration $k$.

In the ZMP procedure ${ }^{26}$ and in the EPI method, ${ }^{25}$ the self-repulsion potential $v^{k}\left[\Delta_{C}^{k}\right]$, appearing in Eq. (5) was defined as

$$
v^{k}\left[\Delta^{k}\right]=-\int d \mathbf{r} \frac{\Delta^{k}}{\mathbf{r}-\mathbf{r}^{\prime}} .
$$

Such potential corresponds to the electric field arising from the density difference $\Delta^{k}$, and introduces a driving force to make $\rho_{\mathrm{A}}^{k}+\rho_{\mathrm{B}}^{k}$ coincide with the reference density $\rho$. However, in this work, it is found that it is more efficient to substitute the Coulomb self-repulsion potential [Eq. (6)] by the exchange self-repulsion potential defined in the atomic basis set representation as a matrix whose elements are given by

$$
v_{i, j}^{k}\left[\Delta^{k}\right]=\frac{1}{2} \sum_{m, l} \Delta_{m, l}^{k}(i m \mid l j),
$$

where $\Delta_{i, j}^{k}$ are the matrix elements of $\Delta^{k}$, and $(i m \mid l j)$ denotes the bielectronic matrix elements among the atomic basis functions.

The iterative procedure is summarized as follows:

1. Initially, the two EPs are set to zero, $\mathcal{V}_{C}^{k=0}=0$. Therefore, the initial densities correspond to the isolated fragments $\mathrm{A}$ and $\mathrm{B}$.

2. The density difference $\Delta^{k}$ is evaluated and with it the exchange self-repulsion potential $v^{k}$ using Eq. (7).

3. The MHF equation is solved for subsystem A for several values of $\lambda$. Choosing the $\lambda$ corresponding to the minimum $\delta_{k}$ value $\lambda_{\min }$, the $\mathrm{EP} \mathcal{V}_{\mathrm{A}}^{k+1}$ is obtained [Eq. (5)] and the MHF [Eq. (4)] is solved for A, determining $\rho_{\mathrm{A}}^{k+1}$.

4. A new density difference is set, $\widetilde{\Delta}^{k}=\rho-\rho_{\mathrm{A}}^{k+1}-\rho_{\mathrm{B}}^{k}$, and with it a new exchange self-repulsion potential, $\widetilde{v}_{i, j}^{k}$, is obtained. 
5. The MHF equation is solved for subsystem B looking for the new $\tilde{\lambda}$ factor, which minimizes $\delta^{k}$. Using this $\tilde{\lambda}_{\min }$ value, the $\mathrm{EP}$ for $\mathrm{B}$ is determined as $\mathcal{V}_{\mathrm{B}}^{k+1}=\mathcal{V}_{\mathrm{B}}^{k}$ $+\lambda \widetilde{v}^{k}\left[\Delta^{k}\right]$ and the MHF equation is solved for $\mathrm{B}$, determining $\rho_{\mathrm{B}}^{k+1}$

6. If the $\delta^{k+1}$ thus obtained is smaller than a given $\epsilon$ the procedure is stopped. If not, another loop is started from point 2. If the error $\delta^{k+1}>\delta^{k}$, the minimization procedure is also finished.

With the $\mathcal{V}_{\mathrm{A}}$ potentials thus determined, the resolution of the MHF equation is performed to obtain the molecular orbitals, the density $\rho_{\mathrm{A}}^{k}$, and the HF energy $E_{\mathrm{HF}}^{\mathrm{A}}$. Thus, the EPs and corresponding partitioned densities are obtained simultaneously.

The density partition is arbitrary, and depends on the method used. In the present iterative case, it also depends on how $\rho$ is calculated, on the initial guess made for $\rho_{\mathrm{A}}^{0}$ and $\rho_{\mathrm{B}}^{0}$ (corresponding to those of the isolated subsystems) and on the self-repulsion potential chosen.

Finally, the orbitals localized in $\mathrm{A}, \phi_{\mathrm{A}, l}^{k}$, are used in a multireference configuration interaction (MRCI) calculation to obtain more accurate electronic energies $E^{\mathrm{A}, \mathrm{CI}}$. The correlation energy is determined as $E_{\mathrm{cor}}^{i=0}=E_{\mathrm{A}, \mathrm{CI}}^{i=0}-E_{\mathrm{HF}, \mathrm{A}}$. The ground state energy of the total system $\mathrm{AB}$, including electronic correlation locally in subsystem $\mathrm{A}$, is then given by

$$
E_{\mathrm{CI}}^{0}=E_{\mathrm{HF}}^{\mathrm{tot}}+E_{\mathrm{cor}}^{i=0}=E_{\mathrm{tot}, \mathrm{HF}}+E_{\mathrm{A}, \mathrm{CI}}^{i=0}-E_{\mathrm{HF}, \mathrm{A}},
$$

where $E_{\mathrm{tot}, \mathrm{HF}}$ is the HF energy obtained for the whole system $\mathrm{AB}$. Thus, when using a DFT method, the correlation potential is not introduced to avoid the double-counting of this term.

The EP used to calculate the correlated subsystem A serves as a constraint to mimic the effects of the environment. This is by itself an approximation, neglecting any dynamical correlation between subsystems A and B, as well as it considers a simple monoelectronic potential term to describe the potential interactions among the two subsystems. In addition to this, it should be mentioned that a different EP would be obtained if $\rho$ were calculated with a higher correlated method.

\section{RESULTS AND DISCUSSION}

Here we shall present calculations on $\left(\mathrm{H}_{2}\right)_{5}$ and $\mathrm{H}_{2}$ $+\left(\mathrm{H}_{2}\right)_{5}$, as done in Ref. 25, to check the present DDEPI method. All the calculations are performed using the MOLPRO package. ${ }^{35}$ The details of the calculations can be found in Ref. 25.

\section{A. $\mathrm{H}_{10}$ chain}

To show how the method works, it is first applied to $\mathrm{H}_{10}$ with an equidistant internuclear distance of 1.3 a.u. The chain is placed parallel to the $\mathrm{z}$-axis with the origin at the center of mass of the system. Subsystem A is formed by the two central hydrogen atoms, at $\mathrm{Z}= \pm 0.65$ a.u., while $\mathrm{B}$ is composed by the remaining eight atoms.

As described above, the iterative DDEPI method starts with HF calculations of the total system and subsystems A

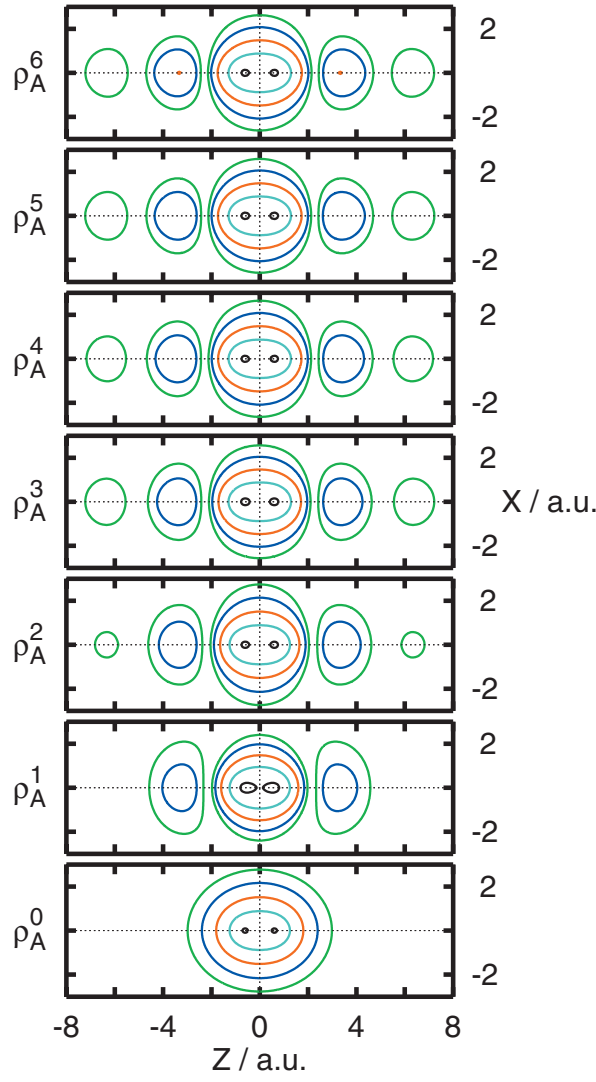

FIG. 1. Contour plot of the $\rho_{\mathrm{A}}^{k}$ for different iterations. The contours are 0.4, $0.1,0.025,0.006$, and $0.0015 a_{0}^{-3}$. These densities correspond to subsystem A, formed by the two central hydrogen atoms of the $\mathrm{H}_{10}$ linear chain, placed at $\mathrm{Z}= \pm 0.65$ a.u., with an equidistant internuclear distance of 1.3 a.u.

and $\mathrm{B}$, with the initial embedding potential set equal to zero. With these three initial densities, the first iteration is started by determining $\mathcal{V}_{\mathrm{A}}^{k=1}$. The $\rho_{\mathrm{A}}^{k}$ densities obtained for subsystem A along the iterative procedure are shown in Fig. 1. Initially, $\rho_{\mathrm{A}}^{k=0}$ is well localized in the region around the two central atoms, but becomes slightly delocalized over the whole system as the iteration goes on. This is in contrast to what happened with the previous EPI method.

The density difference in Fig. 2 decreases significantly, approaching to zero as the number of iterations increases. This situation constitutes a significant improvement with the previous EPI method, in which the density difference associated to the A subsystem was reduced until a given limit, from which no further reduction could be achieved (see Fig. 4 of Ref. 25). Clearly this problem can be attributed to the lack of v-representability of the initial reference $\rho_{\mathrm{A}}$ density used in the EPI method, which was obtained by a simple Mulliken method. ${ }^{25}$ The present method overcomes this problem because the densities associated to each subsystem are the solution of a MHF equation. Although the convergence of the density difference presents an oscillatory behavior, it can be continued arriving to values of $\delta^{k}$ of the order of $10^{-3}$ a.u. only after eight iterations, while initially was 3.7 a.u.

The present DDEPI method divides the total density among the two subsystems, yielding a good convergence. It depends on the initial densities considered for A and B, and on the self-repulsion potential used. In the present case, us- 


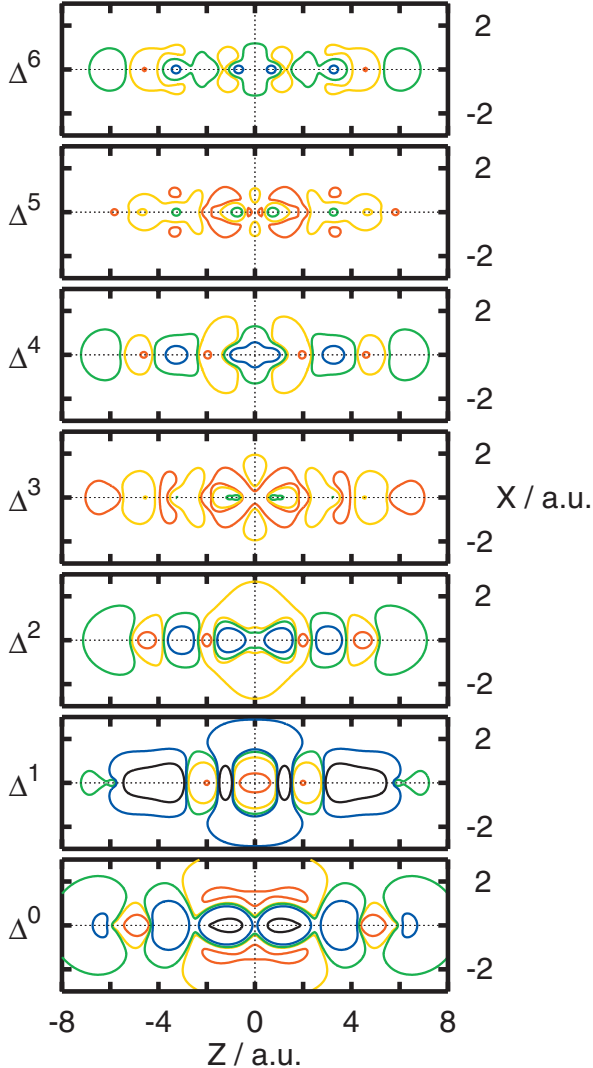

FIG. 2. Density difference $\Delta^{k}$, obtained for $\left(\mathrm{H}_{2}\right)_{5}$ in the $\mathrm{XZ}$ plane as a function of the iteration, from $k=0$ (bottom panel) corresponding to no EPI, up to $k=6$ (top panel). Contours are: 0.05 (black), 0.005 (blue), 0.0005 (green), -0.0005 (yellow), and -0.005 (red) $a_{0}^{-3}$. See also the legend of Fig. 1 for clarity.

ing HF methods, the exchange self-repulsion potential, Eq. (7), yields better convergence than the coulomb one, Eq. (6), previously used in the EPI method. ${ }^{25}$ Other selfrepulsion potentials can be defined to improve the procedure. In particular, the efficiency of the method decreases when using DFT methods instead of HF. For those cases, it may be interesting to design other self-repulsion potentials to improve the accuracy and efficiency of the method.

The partition procedure yields to v-representable $\rho_{\mathrm{A}}$ and $\rho_{\mathrm{B}}$ densities, as commented above, which are constructed from localized molecular orbitals in each of the two subsystems. These orbitals are used in post-HF methods to include electronic correlation locally in subsystem A, thus reducing the computational effort, as described below. The molecular orbitals thus obtained can also be used to get a physical insight of the effect of the environment on a given subsystem, as the analysis of which orbitals are occupied and how they change with the environment.

The convergence along the iterative procedure of the EP used to partition the density for the $\mathrm{H}_{10}$ case is shown in Fig. 3. It presents a sequence of maxima and minima along the axis of the $\mathrm{H}_{10}$ chain. The convergence of the EP is fast, and the positive maximum at $\mathrm{Z}=0$ clearly produces a repulsive effect in the electronic cloud toward the rest of the chain. This fact, together with the shallow minima appearing on the outer hydrogen atoms, explains why the density $\rho_{\mathrm{A}}$ slightly spreads over the whole chain. This is quite different with the

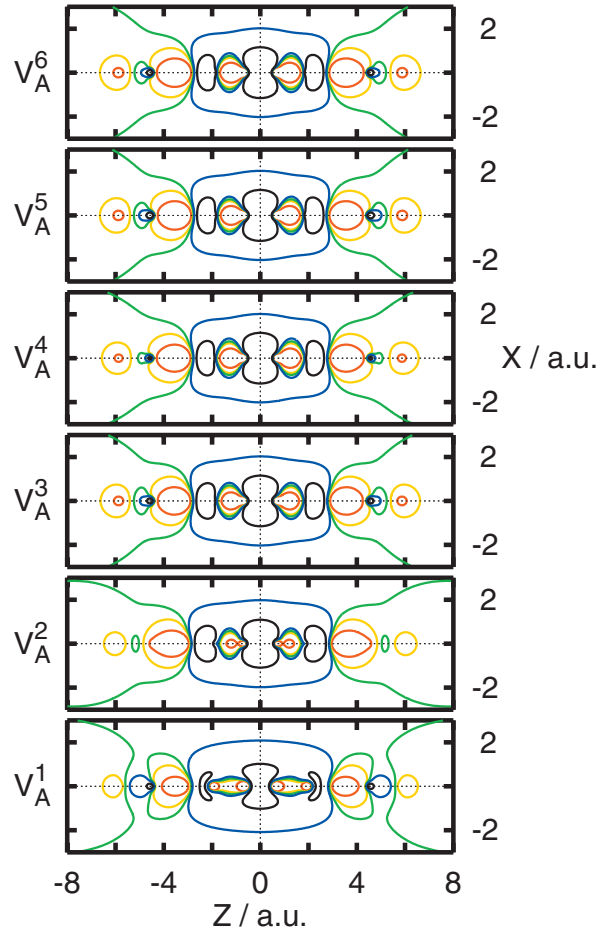

FIG. 3. EP $\mathcal{V}^{k}$, obtained for $\left(\mathrm{H}_{2}\right)_{5}$ as a function of the iteration, from $k=1$ (bottom panel) up to $k=6$ (top panel). Contours are: 0.5 (black), 0.1 (blue), 0.0 (green), -0.1 (yellow), and -0.5 (red) See also the legend of Fig. 1 for clarity.

situation found previously with the EPI method, for which the EP presents a relatively deep well in the A subsystem, and a considerably high barrier out of it, which prevented the density of A to spread over the whole system (see Fig. 5 of Ref. 25)

The next step is the introduction of the electronic correlation using the localized orbitals. Here it has been done at HF and MRCI levels on the A subsystem and on the whole system for comparison, as a function of the distance of the different $\mathrm{H}_{2}$ subsystems within $\mathrm{H}_{10}$ as done previously with the EPI method. ${ }^{25}$ The repulsive barrier at short distances at $\mathrm{HF}$ level is pretty well reproduced when considering only the $\mathrm{H}_{2}$ subsystem within the embedding surrounding $\mathrm{H}_{2}$ subunits, as it was also the case in the EPI method (see Fig. 6 of Ref. 25). At MRCI level, the dynamic correlation among A and $\mathrm{B}$ is introduced, yielding a shallow van der Waals well when considering the whole system. When using a MRCI method on the A subsystem within the DDEPI method, this well is not reproduced since the B subsystem is not introduced, and hence the dynamic correlation among A and B cannot be described. ${ }^{25}$ The van der Waals interaction between a $\mathrm{H}_{2}$ molecule and $\mathrm{H}_{10}$ chain within the DDEPI method is described below.

\section{B. Van der Waals interactions of $\mathbf{H}_{2}$ with a $\mathbf{H}_{10}$ chain}

We consider a $\mathrm{H}_{2}$ molecule (frozen at an internuclear distance of 1.3 a.u.) interacting with the $\mathrm{H}_{10}$ chain, with a distance between adjacent hydrogen atoms of 1.3 a.u. The $\mathrm{H}_{10}$ is in the z-axis with the center of mass at the origin. The $\mathrm{H}_{2}$ is placed parallel to the $\mathrm{y}$-axis with the center of mass along the $\mathrm{x}$-axis at a distance $\mathrm{R}$, which is varied. In the 


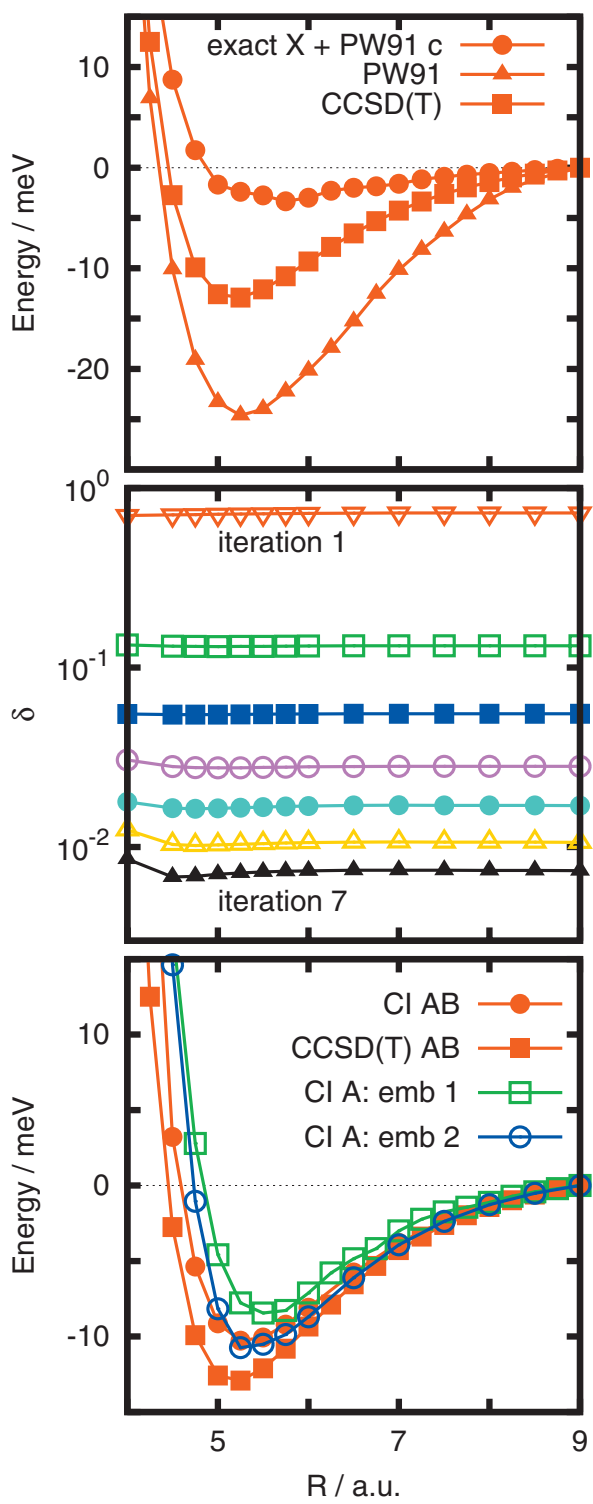

FIG. 4. Comparison of the energies obtained using different methods for $\mathrm{H}_{2}+\mathrm{H}_{10}$ as a function of the distance between the $\mathrm{H}_{2}$ and $\mathrm{H}_{10}$ center of masses. Top panel: $\operatorname{CCSD}(\mathrm{t}), \mathrm{PW} 91$, and exact $\mathrm{X}+\mathrm{PW} 91 \mathrm{c}$ results obtained for the whole system; Middle panel: density difference $\delta^{k}$, obtained with the DDEPI method using as a reference density that obtained with the PW91 method. Bottom panel: energies obtained; full circles (CI $\mathrm{AB}$ ) and full squares (CCSD AB) correspond to a MRCI and $\operatorname{CCSD}(t)$ calculations on the whole system. Open squares (CI A: emb 1) are the MRCI energies obtained with the embedding method of Ref. 25, while open circles (CI A: emb 2) are the corresponding energies of the embedding method presented here.

previous work, ${ }^{25}$ a hybrid exact exchange plus PW91 correlation functional (exact X+PW91c) was used. Using this functional to obtain the electronic density of the whole system $\rho$, the present DDEPI method yields to rather small $\delta$ values in the density partition process. The reference density, however, is not optimal, since the exact $\mathrm{X}+\mathrm{PW} 91 \mathrm{c}$ functional reproduces very poorly the van der Waals interaction, as can be seen in the top panel of Fig. 4. Alternatively, the pure PW91 functional overestimates the van der Waals well by a factor of more than 2 , but the equilibrium distance is reproduced satisfactorily. For this reason in this work we shall use the density obtained with the pure PW91 functional for describing the whole system as the reference density $\rho$.
The subsystem A is composed by the approaching $\mathrm{H}_{2}$ molecule and the two central atoms of the linear $\mathrm{H}_{10}$ chain. Using the pure PW91 density $\rho$, the DDEPI also converges satisfactorily, and in the middle panel of Fig. 4 the evolution of the $\delta^{k}$ parameter, defined in Eq. (3), is shown as a function of the internuclear distance for several iterations. It is clearly seen that the overall density difference decreases with the number of iterations for all distances. However, after iteration 6, the convergence for short distances gets slower. At each iteration, a new contribution to the EP is added, according to Eq. (5). This implies a shift in the total energy, as discussed for the EPI method. ${ }^{25}$ To describe a potential curve, without introducing artificial jumps, it is necessary to consider a similar number of iterations for all the points along the propagation. In this particular case, after six iterations, some of the points for $\mathrm{R}<4$ a.u. start showing some oscillations. The optimal value considered here is to include only six iterations.

The energy curve obtained here with the new DDEPI method (called emb 2) is shown in the lower panel of Fig. 4, and compared to the result obtained with the EPI method of Ref. 25 (called emb 1), both corresponding to subsystem A. As discussed in Ref. 25, when considering this subsystem isolated, the van der Waals interaction gets much weaker, of the order of 1-2 meV with a longer equilibrium distance. When considering the whole system and correlated methods, such as MRCI and coupled cluster with single and double excitations (with triples perturbately) $\operatorname{CCSD}(\mathrm{T})$, a deeper well appears at $\mathrm{R} \approx 5$ a.u., with a well depth of the order of $13 \mathrm{meV}$ for the $\operatorname{CCSD}(\mathrm{T})$. The MRCI result, slightly shallower, demonstrate the difficulty of including all the electronic correlation. Using the Davidson extrapolation, the energy gets closer to the $\operatorname{CCSD}(\mathrm{T})$ result, but not exactly the same (it is not included in the figure to avoid confusion). Moreover, the pure CCSD energy does not show the van der Waals well, which only appears when including perturbatively the triplet excitations. All this demonstrate the difficulty of describing a so weak interaction, even when considering highly correlated methods. It is then remarkable that the DDEPI results presented here are so similar to the MRCI results. This is a result obtained using the localized embedded orbitals of subsystem A to include electronic correlation.

The occupied and first two virtual orbitals are shown in Fig. 5, for $R=5.25$ a.u., for the isolated $A$ subsystem (equivalent to $\phi_{\mathrm{A}, l}^{k=0}$ ), for the A subsystem including the EP (equivalent to $\left.\phi_{\mathrm{A}, l}^{k=6}\right)$, and to the whole system $\mathrm{AB}\left(\phi_{\mathrm{AB}, l}\right)$. For the isolated A subsystem, the two occupied orbitals correspond to a symmetric/antisymmetric superposition of the orbitals located in the approaching $\mathrm{H}_{2}$ molecule, and in the two central atoms of the $\mathrm{H}_{10}$ chain. Since, the rest of the atoms in the chains present a zero charge, the two $\mathrm{H}_{2}$ fragments in subsystem A are equivalent.

In the whole system there are 12 electrons, which occupy the first six orbitals. $\phi_{\mathrm{AB}, l=3,4}$ correspond to a linear superposition of the orbitals located in the approaching $\mathrm{H}_{2}$ molecule, and an orbital with two nodes along the $\mathrm{H}_{10}$ chain, analogous to that found for isolated A subsystem. The rest of the $\phi_{\mathrm{AB}, l}$ orbitals approximately correspond to the $\mathrm{H}_{10}$ chain, 

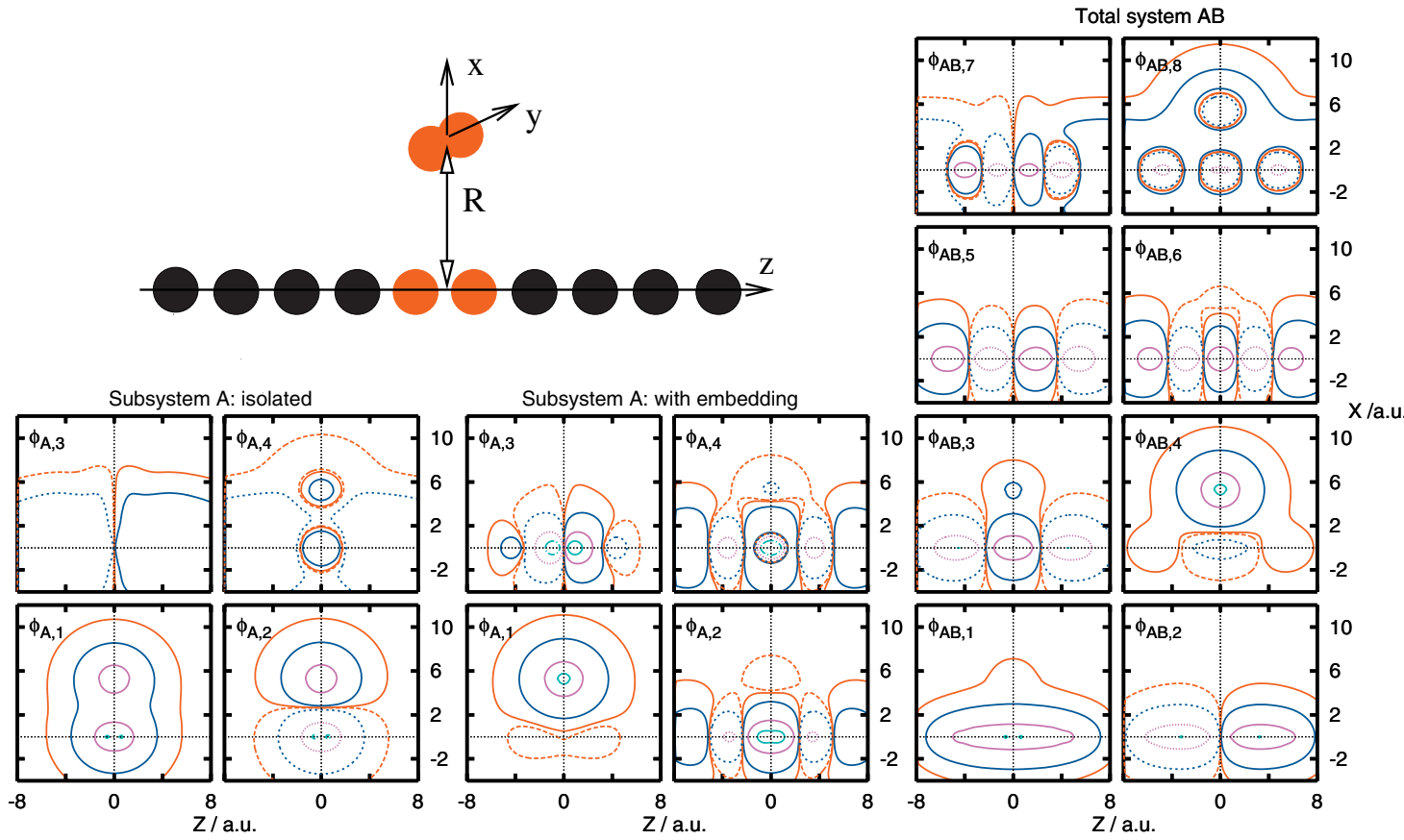

(a)

(b)

(c)

FIG. 5. Contour plot of the amplitude of the first orbitals, in the $\mathrm{XZ}$ plane, at a distance between $\mathrm{H}_{2}$ and $\mathrm{H}_{10}$ of $\mathrm{R}=5.25$ a.u. (in the van der Waals well), obtained for (a) isolated subsystem A (left panels), (b) subsystem A including embedding (middle panels), and (c) total system AB. The contours correspond to $\pm 0.2, \pm 0.1, \pm 0.01$, and \pm 0.001 (solid lines for positive values, dashed lines for negative).

each one showing a particular nodal structure. In all case, the amplitude is homogeneously distributed among all the atoms in the $\mathrm{H}_{10}$ chain.

Finally, when the EP is included in subsystem A, the orbitals (in the middle panels of Fig. 5), show a mixed structure. The two occupied orbitals $\phi_{\mathrm{A}, l=1,2}$ have the same nodal structure than those of orbitals $\phi_{\mathrm{AB}, l=4,6}$ of the whole system. In the embedded case, however, the orbitals are more localized in the central atoms of the $\mathrm{H}_{10}$ chain, as expected. Also, the first virtual orbitals in the embedded subsystem A show a clear analogy with those found for the whole system, but showing this localization effect. This effect is introduced by the EP, since in subsystem A the charges on the noncentral hydrogen atoms are set to zero.

The first virtual orbitals are also localized in the A subsystem, showing tails on the rest of the hydrogen atoms of the $\mathrm{H}_{10}$ chain. This fact explains why the CI using this set of localized orbitals may account for the weak van der Waals interaction. Thus, again the EP plays a fundamental role to properly describe the van der Waals interaction, considering only a subsystem, since the interaction between the two $\mathrm{H}_{2}$ subunits of subsystem A is much weaker, of the order of 1 meV.

\section{CONCLUSIONS}

In this work a new method is proposed to partition the density of a system in two portions, each one being the solution of a Fock equation modified by the inclusion of an EP designed to this aim. The method is first applied to a linear $\mathrm{H}_{10}$ to illustrate how it works. Second, it is applied to describe the $\mathrm{H}_{2}+\mathrm{H}_{10}$ van der Waals interaction by performing highly correlated calculations on a portion of the whole system using localized orbitals obtained as solutions of modified Fock equations.

The partitioning of the density of a system is very frequently done for various purposes: population analysis to build electrostatic models, to rationalize chemical reactivity or to describe long-range electrostatic potentials, ${ }^{36}$ and to separate a system in atoms to make more affordable the calculation of the whole system. ${ }^{12,37-45}$ An extension of this last approach consists of obtaining the density of chemically meaningful fragments, ${ }^{46,47}$ recombining them into new molecules. ${ }^{48}$ For this purpose, the method presented here guaranty the v-representability of each of the densities obtained, and their integrals coincide with the integer number of electrons assigned to each subsystem. In addition, this method also allows obtaining the corresponding localized orbitals in each of the subsystems, which can then be used for different purposes.

As an example, the confined orbitals thus obtained allow the use of highly correlated $a b$ initio methods in subsystem A, to consider electronic correlation in the ground and excited electronic states, as proposed previously. ${ }^{20}$ Also, the confined orbitals obtained feel the presence of the environment and can be used for the understanding of the processes under study.

In this method, the density of the environment is not fixed. This advantage of the present method can be considered as a problem when considering large systems, since the iterative procedure becomes more demanding computationally. This problem can be reduced by dividing the density for one geometry, and afterward use the $\rho_{\mathrm{B}}$ obtained for the rest of the geometries considered. 


\section{ACKNOWLEDGMENTS}

We want to acknowledge Professor T.A. Wesolowski for interesting comments and for sending us several works prior to their publication, and Dr. D. Alcoba for interesting discussions. This work has been supported by CAM (Comunidad Autónoma de Madrid) under Grant No. S-0505/MAT/0303 and by the Ministerio de Ciencia e Innovación under Project Nos. CTQ2007-62898, FIS2007-62006, and CTQ200763332.

${ }^{1}$ S. Humbel, S. Sieber, and K. Morokuma, J. Chem. Phys. 105, 1959 (1996).

${ }^{2}$ M. Svensson, S. Humbel, R. Froese, T. Matsubara, S. Sieber, and K. Morokuma, J. Phys. Chem. 100, 19357 (1996).

${ }^{3}$ H. M. Senn and W. Thiel, Top. Curr. Chem. 268, 173 (2007).

${ }^{4}$ P. Nachtigall, K. D. Jordan, and K. C. Janda, J. Chem. Phys. 95, 8652 (1991).

${ }^{5}$ C. J. Wu and E. A. Carter, Phys. Rev. B 45, 9065 (1992).

${ }^{6}$ R. Konečný and D. J. Doren, J. Chem. Phys. 106, 2426 (1997).

${ }^{7}$ M. P. de Lara-Castells, A. O. Mitrushenkov, O. Roncero, and J. L. Krause, Isr. J. Chem. 45, 59 (2005).

${ }^{8}$ N. W. Winter, R. M. Pitzer, and D. K. Temple, J. Chem. Phys. 87, 2945 (1987).

${ }^{9}$ J. L. Pascual, L. Seijo, and Z. Barandiarán, J. Chem. Phys. 98, 9715 (1993).

${ }^{10}$ V. Puchin, A. Shluger, Y. Nakai, and N. Itoh, Phys. Rev. B 49, 11364 (1994).

${ }^{11}$ E. V. Stefanovich and T. N. Truong, J. Chem. Phys. 102, 5071 (1995).

${ }^{12}$ P. Cortona, Phys. Rev. B 44, 8454 (1991).

${ }^{13}$ E. V. Stefanovich and T. N. Truong, J. Chem. Phys. 104, 2946 (1996).

${ }^{14}$ T. A. Wesolowski and A. Warshel, J. Phys. Chem. 97, 8050 (1993).

${ }^{15}$ J. Neugebauer, M. J. Louwerse, P. Belanzoni, T. A. Wesolowski, and E. J. Baerends, J. Chem. Phys. 123, 114101 (2005).

${ }^{16}$ J. Neugebauer, Ch. R. Jacob, T. A. Wesolowski, and E. J. Baerends, J. Phys. Chem. A 109, 7805 (2005).

${ }^{17}$ Ch. R. Jacob, J. Neugebauer, L. Jensen, and L. Visscher, Phys. Chem. Chem. Phys. 8, 2349 (2006).

${ }^{18}$ T. A. Wesołowski, Phys. Rev. A 77, 012504 (2008).

${ }^{19}$ T. A. Wesolowski, J. Chem. Phys. 106, 7178 (1997).
${ }^{20}$ N. Govind, Y. A. Wang, and E. A. Carter, J. Chem. Phys. 110, 7677 (1999).

${ }^{21}$ T. Klüner, N. Govind, Y. A. Wang, and E. A. Carter, J. Chem. Phys. 116, 42 (2002).

${ }^{22}$ P. Huang and E. A. Carter, J. Chem. Phys. 125, 084102 (2006).

${ }^{23}$ P. Huang and E. A. Carter, Annu. Rev. Phys. Chem. 59, 261 (2008).

${ }^{24}$ C. R. Jacob, S. M. Beyhan, and L. Visscher, J. Chem. Phys. 126, 234116 (2007).

${ }^{25}$ O. Roncero, M. P. de Lara-Castells, P. Villarreal, F. Flores, J. Ortega, M. Paniagua, and A. Aguado, J. Chem. Phys. 129, 184104 (2008).

${ }^{26}$ Q. Zhao, R. C. Morrison, and R. G. Parr, Phys. Rev. A 50, 2138 (1994).

${ }^{27}$ J. D. Talman and W. F. Shadwick, Phys. Rev. A 14, 36 (1976).

${ }^{28}$ C. O. Almbladh and A. C. Pedroza, Phys. Rev. A 29, 2322 (1984).

${ }^{29}$ R. van Leeuwen and E. J. Baerends, Phys. Rev. A 49, 2421 (1994).

${ }^{30}$ O. V. Gritsenko, R. van Leeuwen, and E. J. Baerends, Phys. Rev. A 52, 1870 (1995).

${ }^{31}$ D. J. Tozer, N. C. Handy, and P. Palmieri, Mol. Phys. 91, 567 (1997).

${ }^{32}$ G. K. L. Chan and N. C. Handy, Phys. Rev. A 59, 2670 (1999).

${ }^{33}$ T. Gál, Phys. Rev. A 64, 062503 (2001).

${ }^{34}$ W. Yang and Q. Wu, Phys. Rev. Lett. 89, 143002 (2002).

${ }^{35}$ MOLPRO is a package of ab initio programs designed by H.-J. Werner and P. J. Knowles, and with contributions from J. Almlöf, R. D. Amos, A. Berning, M. J. O. Deegan, F. Eckert, S. T. Elbert, C. Hampel, R. Lindh, W. Meyer, A. Nicklass, K. Peterson, R. Pitzer, A. J. Stone, P. R. Taylor, M. E. Mura, P. Pulay, M. Schütz, H. Stoll, T. Thorsteinsson, and D. L. Cooper, (version 2006).

${ }^{36}$ A. J. Stone, The Theory of Intermolecular Forces (Oxford University Press, New York, 2002).

${ }^{37}$ R. F. W. Bader, Atoms in Molecules: A Quantum Theory (Oxford University Press, Oxford, 1990).

${ }^{38}$ R. G. Gordon and Y. S. Kim, J. Chem. Phys. 56, 3122 (1972).

${ }^{39}$ M. P. Guse, J. Chem. Phys. 75, 828 (1981).

${ }^{40}$ L. Li and R. G. Parr, J. Chem. Phys. 84, 1704 (1986).

${ }^{41}$ W. Yang, Phys. Rev. Lett. 66, 1438 (1991).

${ }^{42}$ W. Yang and T. S. Lee, J. Chem. Phys. 103, 5674 (1995).

${ }^{43}$ M. S. Daw and M. I. Baskes, Phys. Rev. B 29, 6443 (1984).

${ }^{44}$ P. Cortona, Phys. Rev. B 46, 2008 (1992).

${ }^{45}$ T. Zhu, W. Pan, and W. Yang, Phys. Rev. B 53, 12713 (1996).

${ }^{46}$ D. R. Alcoba, A. Torre, R. C. Bochicchio, and L. Lain, Theor. Chem. Acc. 118, 827 (2007).

${ }^{47}$ J. Fernández Rico, R. López, G. Ramírez, I. Ema, and E. V. Ludeña, J. Comput. Chem. 25, 1355 (2004).

${ }^{48}$ T. E. Exner and P. G. Mezey, J. Phys. Chem. A 106, 11791 (2002). 